\title{
Londra e la messinscena delle identità
}

\section{London and the Construction of Identity}

\author{
Cristina Benicchi [c.benicchi@unitus.it] \\ Università degli Studi della Tuscia, Italia
}

\begin{abstract}
RiAsSUNTO
A partire dagli Cinquanta del Novecento per arrivare alle soglie del nuovo millennio, Londra è stata prima approdo di innumerevoli ondate migratorie - originate dalle diaspore successive al progressivo disfacimento dell'Impero coloniale - e poi teatro di un inarrestabile processo di ridefinizione identitaria dello spazio metropolitano e dell'individuo destinati a scardinare le concezioni più tradizionali ed unilaterali del centro e della periferia e a definirsi nella e attraverso la differenze. Partendo da queste considerazioni, il presente studio intende investigare il complesso e articolato processo di formazione e tras-formazione identitaria sopra accennato, attraverso l'analisi di un corpus narrativo della letteratura Black British piuttosto eterogeneo - e non sempre facilmente contenibile all'interno di una definizione come quella di Black British Literature, già di per sé complessa e articolata - ma riconducibile al genere del romanzo di trasformazione, così come definito da Mark Stein. Londra interagisce con i protagonisti, divenendo essa stessa oggetto di trasformazione e protagonista di una articolata e coinvolgente messinscena della formazione identitaria. Fluidità e transitorietà dell'esistenza metropolitana sono lo sfondo dei romanzi di Samuel Selvon, Zadie Smith, Hanif Kureishi, V.S. Naipaul al centro del presente contributo.
\end{abstract}

\section{Parole chiave}

Identità; Città; Transnazionale; Formazione / Trasformazione; Bildung; Postcoloniale

\begin{abstract}
This paper is concerned with those novels - related to black British Literature - that portray the formation, the transformation and the reformation of personal, cultural, ethnic and national identities within London, a urban space that is multiformed, heterotopic and diasporic. London - that is a character and not just the scenery of those novels - suggests an implosive disorder related to the coexistence of immovable dichotomies - such as identity/plurality, freedom/oppression - that frustrate the protagonists' struggle to define their identities and to construct a place to call home. The post-colonial trans-formation novels by Samuel Selvon, Zadie Smith, Hanif Kureishi and V.S. Naipaul - investigated in this study - outline a complex and fragmented process of de-construction and re-construction of identity, whose determining factor is the interaction between the urban space and the individuals. The struggle, performed to be themselves or to find their authentic selves, emphasizes the fluidity of identity, revealing it as almost infinitely changeable, erratic, negotiable and unpredictable.
\end{abstract}

\section{KEYWORDS}

Identity; City; Transnational; Formation / Transformation; Bildung; Postcolonial

RICEVUTO 2015-08-31; ACCETTATO 2015-11-19 
A partire dagli anni Cinquanta del Novecento per arrivare alle soglie del nuovo millennio, Londra è stata prima approdo di innumerevoli ondate migratorie - originate dalle diaspore successive al progressivo disfacimento dell'Impero coloniale britannico - e poi teatro di un inarrestabile processo di ridefinizione identitaria dello spazio metropolitano e dell'individuo, destinato a scardinare le concezioni più tradizionali ed unilaterali del centro e della periferia. Lo spazio metropolitano è sempre più spesso vissuto e raccontato, secondo Homi Bhabha, da coloro che si sono mossi verso il centro dalla periferia; dunque, la marginalità diventa centrale ${ }^{1}$. Partendo da queste considerazioni, il presente studio intende investigare il complesso e articolato processo di formazione e tras-formazione identitaria sopra accennato, attraverso l'analisi di un corpus narrativo tratto dalla cosiddetta letteratura black British. Il corpus è piuttosto eterogeneo - e non sempre facilmente contenibile all'interno di una definizione, come quella di black British Literature, già di per sé complessa e articolata - ma è riconducibile al genere del romanzo di tras-formazione, così come definito da Mark Stein. Lo studioso tedesco definisce tali quei romanzi black British nei quali assistiamo, da un lato, alla formazione dell'individuo e, dall'altro, alla trasformazione e costante maturazione della società britannica. Elementi costitutivi del genere sono:

La costruzione di un luogo da chiamare casa; l'accesso e la liberazione da una storia che è nostra soltanto in parte; gli effetti della migrazione e del dislocamento nelle generazioni successive; la combinazione di tradizioni estetiche differenti e l'interdipendenza di territori culturali diversi; il vessato tema dell'identità (identità personale, culturale, etnica, nazionale); fenomeni di mescolanza e ibridità culturale; differenza culturale e i notori problemi di razzismo; i processi di cambiamento culturale, di creazioni di nuovi spazi, di trasformazione ${ }^{2}$. (Stein 2004: XII)

I romanzi presi in esame racchiudono tutti questi elementi, narrando la formazione e la trasformazione di identità personali, culturali, etniche e nazionali all'interno di uno spazio metropolitano londinese multiforme, eterotopico e diasporico. Tale appare agli occhi di noi lettori la Londra letteraria di Selvon, Naipaul, Smith, Kureishi, scrittori “migranti” o, per meglio dire, eredi della massiccia migrazione che travolse la metropoli londinese a partire dagli anni Cinquanta del Novecento ${ }^{3}$. Nelle loro narrazioni, Londra diventa il simbolo di una realtà ricca di dicotomie, in cui coesistono identità e pluralità, libertà ed oppressione, chiusura ed apertura. La città si fa personaggio, si forma e trasforma al pari degli altri protagonisti che in essa si muovono alla ricerca di se stessi e della propria identità. Il loro girovagare vorticoso, in una Londra che in taluni casi ricorda la "città-ragnatela dove non si danno né percorsi né itinerari né arrivi" (Manferlotti 1995: 302), delinea un complesso e frammentario processo di de-costruzione e ricostruzione, in cui determinante è proprio l'interazione con lo spazio metropolitano, attore, e non semplice spettatore, di una articolata e coinvolgente messinscena della formazione identi-

1 Su questo aspetto si vedano Bhabha (1994) e Hall (1996).

2 La traduzione è dell'autrice. Laddove non ci siano traduzioni italiane edite dei testi citati, le traduzioni si intendono dell'autrice.

3 Il 22 giugno 1948 la SS Empire Windrush, un tempo nave militare tedesca, raggiunge il porto di Tilbury in Inghilterra con a bordo quattrocentonovantadue giamaicani. È l'inizio ufficiale di quel lungo e tormentato processo di immigrazione definito dalla poetessa giamaicana Louise Bennett una colonizzazione inversa (1986: 32). 
taria. Di questa messinscena, il presente contributo intende delineare gli aspetti più significativi, muovendo anche dalla prospettiva offerta da Albertazzi nel riscoprire il dubbio amletico di Jacques Prévert, dell'“essere dove non si è", che lega imprescindibilmente al luogo la definizione di sé, qualunque siano le ragioni della dislocazione dell'io e, dunque, il suo legame con il luogo $^{4}$. Nel palcoscenico metropolitano, dove tutto è caos e la quotidianità scandita da irriducibili pluralismi, i personaggi/individui tracciano percorsi complessi, intricati, eppure capaci di interagire con l'aggrovigliata matassa urbana fino al punto di dipanarla, renderla un luogo familiare, conoscerla, ma soprattutto riconoscersi in quel luogo, come individui dall'identità fluida e dunque infinitamente mutevole, erratica, negoziabile e imprevedibile.

In un'intervista rilasciata allo studioso tedesco Mark Stein, lo scrittore guyanese David Dabydeen afferma: "La condizione moderna è quella dell'erranza. Noi siamo qui per restare ma siamo anche qui per muoverci se necessario, ed emigrare e tornare nei Caraibi e andare da qualsiasi altra parte con i nostri passaporti britannici" (Stein 1999: 8). Il movimento ininterrotto cui l'uomo moderno sembra destinato appartiene ad un ciclo di tras-formazione e rigenerazione, innescato dai flussi migratori e perpetrato dalla realtà metropolitana, che ne costituisce l'approdo. Meta indiscussa delle migrazioni moderne e contemporanee, "con i suoi dettagli quotidiani, il suo misto di storie, lingue e culture, il suo insieme di tendenze globali e distinzioni locali la metropoli contemporanea, è per molti la metafora preferita dell'esperienza del mondo moderno", è "una realtà multiforme, eterotopica, diasporica" che "suggerisce un disordine implosivo, talvolta liberatorio, spesso sconcertante" (Chambers 2003: 105-106).

In questa prospettiva antropologica, Iain Chambers rappresenta un disordine metropolitano niente affatto distruttivo, ma piuttosto stimolo per la produzione di nuovi percorsi dell'immaginazione verso direzioni "precedentemente impensate" ed inesplorate, o verso quell'immenso caos globale, governato dalla fluidità e dalla transitorietà identitarie, magistralmente descritto da Édouard Glissant:

Chiamo caos-mondo - l'ho detto più volte - lo choc, l'intreccio, le repulsioni, le attrazioni, le connivenze, le opposizioni, i conflitti fra le culture dei popoli, nella totalità-mondo contemporanea. [...] si tratta di una mescolanza culturale, che non è semplicemente un melting-pot, attraverso cui al totalità mondo è realizzata. (1998: 62)

In mezzo a questo caos, la città accoglie "quegli incontri, quei meticciati, quelle fusioni, che permettono che gli "altri”, non debbano più essere rappresentati solo ed esclusivamente secondo le nostre onnipotenti categorie scientifiche" e diviene pertanto "luogo privilegiato per l'elaborazione individuale e collettiva della cultura” (Callari Galli 1996: 4), poiché nonostante il trascorrere del tempo e la sua connaturata mutevolezza, la città non smarrisce mai "la sua ragione suprema, vale a dire simultaneità ed incontro" ma, "diventa ciò che è sempre stato: un luogo di desiderio, di permanente squilibrio, sede della dissoluzione delle normalità e delle coercizioni, il momento del gioco e dell'imprevedibile" (Lefebvre 1996: 129).

La trasposizione letteraria di Londra degli autori qui affrontati, pur nella sua eterogeneità, immortala queste complesse dinamiche sociali, rendendo la città palcoscenico di una messinscena, 
in cui l'individuo partecipa "ad una vita sociale e culturale non più data una volta per tutte ma inventata mille volte, giorno dopo giorno"s (Lefebvre 1996: 106).

Narrare la città, per questi autori, significa narrare migrazioni, dislocazioni, ibridazioni, creolizzazioni. È il racconto di una bildung, di una tensione spirituale dell'io che entra in contatto profondamente con il luogo metropolitano e le sue sfere culturali, de-costruendosi e ri-costruendosi, fino a raggiungere una nuova e rassicurante consapevolezza, per cui, sostiene Stuart Hall, "l'identità culturale non è affatto [...] fissa [...] definita dall’essenza o dalla purezza, ma dal riconoscimento di un'eterogeneità e di una diversità necessarie” (1990: 235). La coscienza della dimensione fluida e transitoria dell'esistenza è indubbiamente sollecitata da una città percepita come transforming, translating e transnational.

La città - ricorda Stein - è al contempo transforming / trasformante - in quanto agisce sugli individui trasformandoli - e transformed / trasformata - poiché si lascia a sua volta trasformare da chi la vive. È translating / traducente ed esercita la sua azione traduttoria sul piano del movimento e non, o almeno non esclusivamente linguistico-comunicativo. Il carattere traduttorio della città, d'altra parte, non giunge inaspettato. George Lamming - figura di rilievo della letteratura caraibica anglofona e del mondo accademico, esponente insieme a Samuel Selvon della Windrush Generation, sicuro punto riferimento anche per gli scrittori presi in esame - nel suo capolavoro The Pleasures of Exile descrive l'arrivo di Calibano a Londra come un processo di "traduzione sempre aperta" (1984: 15) di lingua, cultura e tradizione. E Salman Rushdie, nelle sue Imaginary Homelands, definisce tradotta la sua stessa identità:

[...] la parola traduzione deriva, etimologicamente, dal latino "portare di là". Poiché noi siamo persone portate di là nel mondo, siamo individui tradotti. Si ritiene solitamente che qualcosa dell'originale si perda in una traduzione; insisto sul fatto che si possa anche guadagnare qualcosa. (1991: 22)

Gli scrittori migranti/diasporici - afferma Hall - "devono imparare ad abitare almeno due identità, parlare due linguaggi culturali, tradurre e negoziare tra loro" (Hall - Held - McGrew 1992: 310), ragion per cui - secondo Woodcock - rimangono "lavori incompiuti, che seminano il cambiamento nella cultura in cui entrano così come sono cambiati dalla stessa" (Woodcock 1999: 59).

La città, infatti, accoglie culture ed individui in uno spazio fluido e mutevole in cui tutte le componenti sono soggette al cambiamento, in virtù di una reciprocità traduttologica per cui la cultura che traduce è a sua volta tradotta dall'incontro con l'alterità e lo stesso processo attivo e passivo è svolto e subito dalla metropoli transnational / transnazionale.

Nel suo studio sulle rappresentazioni letterarie londinesi, John Clement Ball sostiene che la Londra transnational / transnazionale ha superato le dicotomie linguistiche, locale/globale o nazionale/multinazionale:

Londra come scenario della narrativa postcoloniale è 'transnazionale' per via dei modi unici con cui confonde i binari globale-locale e accoglie nuove forme e narrazioni di identità relazionale, 
e anche per via della sua capacità di includere persone dalle diverse nazioni e diaspore. Questi residenti sono fisicamente distaccati ma psicologicamente e culturalmente attaccati ai luoghi e alle identità nazionali non-inglesi, attraverso i quali sono costantemente esaminate le loro esperienze e rappresentazioni di Londra. La loro città è una comunità transnazionale immaginata che gli scrittori - sia che la immaginino come un prospetto lontano o una realtà attuale - possono incoraggiare gli altri a 'leggere' o vedere come tale. (2004: 31)

Dunque, Londra è immortalata da uno sguardo fisicamente distante, ma idealmente radicato nei luoghi e nelle identità considerati convenzionalmente lontani dalla Britishness, a cui invece appartengono. "Scaturisce", per dirla con le parole di Fusillo, "da una continua tensione fra il locale e l'universale - quella zona ibrida che oggi chiamiamo glocal - fra la singola esperienza individuale e un ampio contesto di sguardi incrociati" (2014: 15). Londra è il "cuore della nostra storia" (1981: 76) - dichiara un'anonima voce della poesia di Walcott The Bright Field - e, in quanto tale, a lei è rivolto l'immaginario dello scrittore che la elegge ad approdo della sua erranza reale o spirituale.

Si tratta, perciò, di una città vissuta simultaneamente come place e come space. Ciò che inizia come uno spazio indifferenziato diventa un luogo, man mano che iniziamo a conoscerlo meglio e gli attribuiamo un valore (Tuan 1977). John Clement Ball a tal proposito afferma:

Paradossalmente, un migrante del Commonwealth che arriva a Londra per la prima volta può aspettarsi di incontrare la città sia come spazio che come luogo. Essa sarà uno spazio in quanto risulterà inconsueta e non ancora segnata dall'esperienza personale delle sue strade e dei suoi edifici. [...] In un altro senso, il nuovo arrivato viene in una città che è sin troppo un luogo: stratificata di valori acquisiti, significati, e immagini che si sono accumulate su 'Londra' nel corso dei secoli di storia imperiale, culturale e letteraria. (2004: 32-33)

Animati dal desiderio di trasformare lo spazio in luogo e dunque di "abitare una Londra transnazionale e postcoloniale reale anziché immaginaria” (Ball 2004: 33), i personaggi letterari talvolta subiscono la frustrazione della disillusione - così è per i londinesi solitari di Selvon, eppure capaci di agire e reagire, o per il narratore alter ego di Naipaul, che vive la trasformazione della città da utopia a distopia - talaltra accettano la fluidità e la transitorietà come elementi essenziali ed inalienabili della loro identità. È questo il caso dei personaggi letterari di Kureishi e Smith.

In The Enigma of Arrival - romanzo del 1987 dalla forte connotazione autobiografica - Naipaul racconta le vicissitudini di un giovane indiano giunto in Inghilterra da Trinidad negli anni Cinquanta e ritrovatosi scrittore proprio lì dopo diversi anni. Al termine delle peregrinazioni londinesi lo scrittore, "definito dalle sue scoperte letterarie e dalle sue idee" (Naipaul 1988: 345), percepisce il declino di Londra e la disillusione di un'immagine del "cuore dell'Impero" che per anni aveva custodito invariata dentro la sua mente. Dal mito e dagli stereotipi letterari alla città reale, le immagini si susseguono:

Mi ero fatto un'idea così grandiosa della città quando ero arrivato! Ma la grandiosità l'avevo portata io giungendovi. [...] Così giunsi a convincermi che la grandezza era cosa del passato; 
che ero arrivato in Inghilterra nell'epoca sbagliata, troppo tardi per trovarvi il cuore dell'Impero che io (da provinciale proveniente da un remoto angolo di questo stesso Impero) mi ero creato nella fantasia. (1988: 133)

L'Inghilterra sognata e immaginata si rivela un'illusione che pone la città di Londra al di fuori del tempo e dello spazio, in una dimensione altra:

Ero dunque pronto ad accettare che il mondo nel quale mi ero ritrovato a Londra fosse diverso e da meno rispetto al mondo perfetto che avevo sognato. A Trinidad, fanciullo, avevo collocato questo mondo lontano nello spazio, forse a Londra. Una volta a Londra, situavo questo mondo perfetto lontano nel tempo, in un tempo precedente. (1988: 135)

La Londra conosciuta solo attraverso la letteratura si rivela distante da quella vissuta:

La Londra che conoscevo e che possedevo nell'immaginazione era la Londra di Dickens. Era stato Dickens - e i suoi illustratori - a darmi l'illusione di conoscere la città. Io ero dunque, senza rendermene conto, come quei russi di cui avrei saputo (con mia gran meraviglia) che ancora credevano esistesse la Londra di Dickens. (1988: 136)

La metropoli londinese di Naipaul è un città mutevole, sottoposta all'invasione di "tutte le genti barbare del globo" e destinata a perdere l'univocità nazionale per trasformarsi in una capitale del mondo:

Nel 1950 a Londra assistevo all'inizio di quel grande movimento di popoli che avrebbe caratterizzato la seconda metà del ventesimo secolo [...] Le città come Londra erano destinate a cambiare. Avrebbero cessato di essere città più o meno nazionali per divenire capitali del mondo, tante Rome moderne, il modello di come sarebbero dovute essere le metropoli agli occhi degli isolani come me e di genti ancora più differenti per lingua e cultura. Città in cui sarebbero accorsi in cerca di cultura, di articoli di pregio, di modi eleganti, di libertà, tutte le genti barbare del globo, le genti della foresta e del deserto, Arabi, Africani, Malesi. (1988: 144-145)

La mancanza di corrispondenza tra la Londra immaginata e quella vissuta genera in Naipaul un profondo senso di disagio sociale ed individuale. Apparentemente affascinato dalla mescolanza metropolitana, egli in realtà sembra prendere le distanze dai barbari invasori di Londra e da una città che, paradossalmente, dopo essere stata vissuta e sperimentata, è estranea, ignota, enigmatica, alienante al punto da emarginare radicalmente l'individuo, che sopraffatto da tanta estraneità riduce il suo spazio vitale fino ad annullarlo completamente:

Venni a Londra. Era diventata il centro del mio mondo, avevo lavorato duramente per venirci. E mi trovai sperso. Londra non era il centro del mio mondo. Ero stato tratto in inganno; ma dove altro andare. Era un buon posto per perdercisi, una città esplorata dal cuore neutrale verso l'esterno finché, dopo anni, si definiva in una babele di radure separate da tratti di ignoto in cui erano incisi sentieri esilissimi. Diventai solo l'abitante di una metropoli, derubato di ogni legame, 
col tempo che passava e mi portava via da ciò che ero, rigettato sempre più dentro me stesso, a lottare per mantenere l'equilibrio e tener vivo il pensiero del mondo chiaro di là dai mattoni e l'asfalto e il caos delle linee ferroviarie. Le terre mitiche svanivano, e nella metropoli ero confinato in un mondo d'una piccolezza mai conosciuta. Diventai il mio appartamento, il mio scrittorio, il mio nome. (Naipaul 1999: 49-50)

L'esplosione della vitalità metropolitana si scontra con l'inerzia di un io incapace di comunicare, di relazionarsi, completamente imploso in se stesso e imprigionato in una vita di inesorabile solitudine.

Allo stesso modo di Naipaul anche Selvon - come lui giunto a Londra negli anni Cinquanta, Naipaul arriva esattamente nel 1950 - racconta con straordinaria maturità linguistica, le difficoltà dei suoi protagonisti, esaltandone al tempo stesso la vitalità, l'inventiva e la capacità di sopravvivenza in una realtà metropolitana sopraffatta dalla solitudine. The Lonely Londoners è il primo romanzo - e l'unico ad essere stato tradotto in italiano col titolo di Londinesi solitari - di una trilogia, ultimata nell'arco di trent'anni da Moses Ascending (1975) e Moses Migrating (1983), in cui le vicende di un gruppo di immigrati caraibici a Londra ruotano attorno alla figura carismatica del saggio Moses.

Nella trilogia di Selvon, la collocazione urbana dei personaggi è rivelatrice della loro stessa condizione sociale ed esistenziale. "Sepolti lontani dal chiassoso baccano della metropoli" (Sandhu 2003: 160), i londinesi di Selvon sono esclusi dalla vitalità del centro metropolitano e si ritrovano a vivere nei seminterrati di Bayswater o Notting Hill. In particolare, i diversi spazi abitativi occupati da Moses nella trilogia raccontano formazione e tras-formazione dell'individuo e della realtà sociale urbana in cui egli si muove, immortalando momenti diversi della diaspora originata dal progressivo crollo dell'Impero coloniale britannico. Da questo punto di vista, la trilogia di Selvon si rivela esplorazione della disillusione dell'individuo diasporico Moses questo rappresenta - che vede progressivamente svanire speranze e aspettative legate al suo distacco dalla terra madre. In The Lonely Londoners, Moses è "chiuso, in quella piccola stanza con Londra e la vita al di fuori” (1956: 167), in Moses Ascending, confessa la necessità ineluttabile di cambiare casa: "Avevo la sensazione che se non avessi fatto un passo adesso, sarei stato destinato alla brigata del seminterrato per il resto della mia vita" (1975: 9). E in effetti, proprio nel secondo volume della trilogia, Moses si trasferisce ai piani alti, per così dire, acquistando un decadente complesso di appartamenti a Shepherd's Bush e occupandone l'attico, in qualità di proprietario. La nuova condizione, tuttavia, non avrà lunga durata e ben presto Moses si ritrova costretto ad abbandonare l'attico per il seminterrato. In Moses Migrating, il racconto si apre otto anni più tardi rispetto alla conclusione del volume precedente, con Moses pronto a partire per Trinidad e indeciso se questo ritorno a casa sia una semplice visita per celebrare il Carnevale o piuttosto una relocation permanente. Dal momento che a questa partenza, probabilmente, seguirà un ritorno, Moses ha comunque deciso di mantenere la proprietà del complesso di appartamenti, affidandone la gestione all'amico Galahad. Moses si trova così a rivivere il viaggio di ritorno a Trinidad, come anni prima aveva vissuto quello verso l'Inghilterra. Oggi come allora, la sua cabina è vicina alla sala macchine. Giunto a Trinidad non c’è una casa vera e propria ad attenderlo, ma un albergo. Prende la sua residenza al "de Hilton", dove, non solo sembra riproporsi una struttura sociale classista, ma Moses vive con difficoltà l'esperienza della 
relocation e di cosa sia realmente la casa/home e dunque il suo significato più intimo e profondo. Non trovando risposte ai propri dubbi e non potendo considerare nessun luogo come casa/ home, Moses si prefigge un obiettivo: quello di raccontare, o meglio spiegare, la casa/home che ha appena lasciato, Londra, all'altra casa/home, abbandonata venticinque anni prima. Moses, in realtà, è convinto che difficilmente potrà far comprendere l'Inghilterra ai suoi conterranei, ma nel suo tentativo c'è comunque una forte volontà di affermazione identitaria di chi, probabilmente, non si sente affatto privo di radici, o di senso di appartenenza, ma è convinto di aver cercato, sebbene invano, di metter radici ovunque abbia vissuto. Il passaggio da black immigrant a black londoner (Ackroyd 2000: 718) passa attraverso l'acquisto della casa, ma non rappresenta un approdo definitivo, perché la vicenda di Moses si evolve nel tempo, rispecchiando le vicissitudini storiche e sociali che, se in The Lonely Londoners sono strettamente legate alla diaspora caraibica - quando Londra rimane, comunque, una "triste città solitaria" dove "nessuno ti accetta per davvero. Ti tollerano, sì, ma non puoi andare nelle loro case a mangiare o a sederti e chiacchierare. Non cè nessun genere di famiglia" (Selvon 1956: 149). "Londra è un posto così. È divisa in piccoli mondi, e tu te ne stai nel mondo a cui appartieni e non sai niente di quello che capita negli altri" (Selvon 1956: 82) - in Moses Ascending rispecchiano le nuove non promettenti condizioni dei neri in Gran Bretagna e in Moses Migrating richiamano addirittura i temi di una globalizzazione ancora primordiale e soltanto accennata, ma comunque significativa.

Diversamente da Moses - spesso semplice destinatario dei racconti di peregrinazioni metropolitane degli amici - Karim, il giovane indiano protagonista di The Buddha of Suburbia - romanzo del 1990 - di Hanif Kureishi, girovaga per la città in cerca di un'identità che ritiene di poter trovare nel centro urbano, lontano dalla periferia. Ma già nell'incipit del racconto, il lettore si trova di fronte ad un personaggio assolutamente mutevole, uno "strano miscuglio" come egli stesso si definisce:

Mi chiamo Karim Amir e sono un vero inglese, più o meno [sono quasi un vero inglese $]^{6}$. La gente mi considera uno strano tipo di inglese, come se appartenessi a una nuova razza, dal momento che sono nato dall'incrocio di due vecchie culture. A me però non importa, sono inglese (non che la circostanza mi riempia di orgoglio), vengo dalla periferia a sud di Londra e sto andando da qualche parte. Forse è stato lo strano miscuglio di continenti e sangue, un pezzo qui e uno là, l'avere un senso di appartenenza e il non averlo, a rendermi una persona irrequieta, che tende ad annoiarsi facilmente. (2003: 7)

L'incertezza di Karim nel definirsi un "vero inglese”, superbamente sottolineata, da quell'avverbio "almost", "quasi/ più o meno" è piuttosto la certezza di esser destinato ad un'esistenza fluida, che gli consentirà di trovare una via nel mondo (Naipaul 1994) e vivere attraverso piuttosto che nonostante le differenze. Una consapevolezza che permea la Londra di Kureishi nella sua totalità, laddove anche il matrimonio finale tra l'indiano Haroon e l'inglese Eva lascia presagire una nuova società, più consapevole del processo di negoziazione identitaria e disposta ad accettare sia l'erosione di nozioni univoche della Britishness che il conseguente approdo al multiculturalismo. Nulla di tutto questo è presente nel primo matrimonio di Haroon con la madre di 
Karim, Margaret. Ella, infatti, è sì inglese, ma il suo modo di agire denota staticità, incapacità di affrancarsi dal vecchio e da opprimenti tradizioni. Margaret nasconde il suo corpo sotto innumerevoli strati di vestiti e la sua personalità è intrappolata nel ruolo tradizionale della casalinga intenta a farsi carico di tutti i lavori domestici e custode delle tradizioni. Eva, invece, è quanto di più distante dai conformismi della tradizione. Inglese anche lei, ma assolutamente esotica nell'aspetto, è dinamica e disinibita e rappresenta la novità, la gioia di vivere, l'eccitazione e la libertà. Da questo punto di vista, quindi, è lecito credere che nel matrimonio tra Haroon ed Eva - contraddistinto da tanta passione e libertà - Kureishi voglia immortalare l’opportunità, tanto agognata da Haroon, di agire e cambiare la propria realtà, fuoriuscendo da una condizione esistenziale oppressa da tradizioni e univoche categorie identitarie ormai desuete.

Di questa nuova Londra, consapevolmente multi-etnica, Zadie Smith - scrittrice anglo-giamaicana - offre una vivida rappresentazione nel suo primo romanzo: White Teeth, pubblicato nel 2000, e annoverato da Mark Stein come il romanzo di tras-formazione per antonomasia per il modo in cui racchiude gli elementi costitutivi del genere.

Epopea sulla Gran Bretagna multietnica, il romanzo di Smith deve la capacità di condensare tutti questi elementi alla pluralità di voci e prospettive che animano il racconto delle vicissitudini di tre famiglie, sì londinesi, ma appartenenti a diversi retroterra etnici, culturali e religiosi: i Chalfen (ebrei), i Joneses, (giamaicani), gli Iqbals (bangladesi). La trasversalità geografica della narrazione lega i personaggi all'India, al Bangladesh, ai Caraibi e, con un accenno alla Seconda Guerra Mondiale, anche alla Bulgaria e all'Italia. Il tempo stesso sembra subire il ritmo trasversale della dislocazione geografica laddove il racconto si fa multigenerazionale e le passate esperienze metropolitane dei genitori si confondono con quelle più recenti delle nuove generazioni.

Franco Marcoaldi su Repubblica scrive: "Lobiettivo della Smith è restituirci la complicata, paradossale rete di rapporti che si stabilisce nell'universo multietnico e multireligioso della Londra anni '70 e '80, con una popolazione di immigrati di prima e seconda generazione che oscilla tra assimilazione e nostalgia”. La critica ha riconosciuto il contributo di Zadie Smith alla raffigurazione etnica di Londra, spesso attribuito a scrittori come Selvon e Kureishi, piuttosto che a scrittrici. Pilar Cuder-Dominguez dedica il suo saggio "Ethnic Cartographies of London in Bernardine Evaristo and Zadie Smith" (2004) a quelle scrittrici come Monica Ali, Evaristo e Smith che "dipingono il cuore della Englishness come una città multietnica e multiculturale" (2004: 45) e Itala Vivan definisce White Teeeth uno "splendido esercizio di costruzione dell'identità" (2002: 32).

$\mathrm{Ne}$ consegue un intreccio ricco di figure e vicende, tenute insieme dall'amicizia ultradecennale tra l'inglese Archie - impiegato in una tipografia - e il bangladese Samad - capocameriere in un ristorante indiano - compagni di sventura nella Seconda Guerra Mondiale e poi casuali compagni di casa nel nordovest di Londra.

A differenza dei racconti di Selvon e Naipaul, dall'impianto fortemente autobiografico, White Teeth, afferma Smith in un'intervista all'Observer: "non si basa sulla mia personale esperienza familiare. [...] Gran parte del mio lavoro è frutto di supposizioni o deriva dalla lettura dei resoconti degli immigrati arrivati in Gran Bretagna”. Inevitabile, tuttavia, ritrovare molto dell'autrice nel personaggio di Irie.

I retroscena familiari dei protagonisti di White Teeth rivelano individualità britanniche niente affatto monolitiche, ma forti di una molteplicità culturale ed etnica derivata da un passato di intrecci e diaspore, che la città ha saputo fagocitare e che risultano endemiche alla nozione 
stessa di Britishness: nessuna purezza, dunque, se non inventata, questo il punto di partenza del romanzo. In questo modo, scopriamo che anche i Chalfen - in apparenza i meno contaminati dal multiculturalismo londinese e perciò degni rappresentanti della classe media bianca inglese - sono, in realtà, parte integrante di una società metropolitana inesorabilmente sincretica. Marcus Chalfen, infatti, è giunto in Inghilterra in conseguenza della diaspora ebraica e suo figlio è: "[...] un'impollinazione incrociata (cross-pollination) tra una orticoltrice femminista cattolica non osservante ed un intellettuale ebreo [...]” (2000: 321). Eppure, anche nella Londra di Smith, non mancano le reticenze di chi - tanto tra i personaggi white British quanto tra i black British - stenta ad accettare la "mescolanza culturale e sociale, il cambiamento continuo" (Ball 2004: XI-XII). Reticenze inaccettabili, perché anacronistiche, lascia intuire Smith. Anacronistica risulta, infatti, l'incapacità di Samad di comprendere i propri figli e le loro scelte in una società sempre più intricata e creolizzata. Dalle sue parole - testimoni, peraltro, di un vero scontro generazionale - emerge la delusione di chi, entrato in un paese straniero, è guardato costantemente con sospetto:

In questi giorni ho la sensazione che quando si entra in questo paese si fa un patto con il diavolo. Si consegna il passaporto, si riceve un timbro, si vuole guadagnare qualcosa, si comincia... ma allo stesso tempo si vuole tornare indietro! E chi vorrebbe mai restare? Freddo, umido, miseria; cibo orribile, giornali spaventosi... e chi vorrebbe mai restare? In un posto dove non si è mai benaccetti, ma solo tollerati. Appena tollerati. Come se si fosse degli animali diventati finalmente domestici. Chi vorrebbe mai restare? Ma si è stretto un patto con il diavolo... ti trascina dentro e all'improvviso non sei più adatto al ritorno, i tuoi figli diventano irriconoscibili, non appartieni più a nessun posto. (Smith 2000: 330)

Alle parole di Samad, fanno eco quelle di un narratore che, con vivida lucidità, immortala la dilagante e inarrestabile fluidità culturale della contemporaneità che, per quanto ancora difficile da ammettere, non può certamente essere ignorata:

Questo è stato il secolo degli sconosciuti, di pelle scura, gialla e bianca. Questo è stato il secolo della grande sperimentazione immigratoria. [...] Ragazzi con il nome di battesimo e il patronimico in rotta di collisione. Nomi che al loro interno celano esodi di massa, barche e aerei stracolmi, sbarchi gelidi, controlli medici. [...] Eppure, malgrado tutte le mescolanze, malgrado il fatto che siamo scivolati con ragionevole facilità gli uni nelle vite degli altri (come chi torna nel letto dell'innamorata dopo una passeggiata notturna), malgrado tutto questo, è ancora difficile ammettere che non cè nessuno più inglese degli indiani, [nessuno più indiano degli inglesi $]^{7}$. (Smith 2000: 336-337)

Smith traduce l'indeterminatezza imprevedibile della metropoli e di chi la abita, raccontando l'inesistenza di uno spazio incontaminato e definito, che può costantemente essere "trasformato in modi inaspettati da scelte, combinazioni, casualità, o avvenimenti imprevisti” (Ball 2004: 243). La sua è l’immagine della metropoli delineata da J.C. Ball, che "sradica le persone e le ra-

7 La frase in corsivo tra parentesi quadre è stata aggiunta dall'autrice perché non compare nelledizione Mondadori. 
duna con i loro mondi affinché si incontrino le une con le altre, crea nuovi grovigli in aggiunta a quelli vecchi, e produce nuove mescolanze e identità. Sempre imprevista e pronta a trasformare, la metropoli non può mai essere uno spazio neutrale e finale" (Ball 2004: 244). I suoi abitanti, lucidamente coscienti dell'essere il frutto di un processo di cross-pollination, sono anche altrettanto consapevoli del coinvolgimento che li lega gli uni agli altri ed ostentano sicurezza nell'affermare, senza timori, questa loro condizione: "Siamo tutti coinvolti gli uni con gli altri" (Smith 2000: 376) - afferma Alsana Iqbal - "Siamo contaminati gli uni dagli altri” (Smith 2000: 354). Irie Jones, rappresentante della nuova generazione, accetta con serenità la sua ibridità $\mathrm{e}$ come lei anche i suoi contemporanei non sono affatto turbati dall'essere ciò che sono, "qualcosa di ibrido" (Smith 2000: 231).

In conclusione, le narrazioni di Londra prese in esame mostrano come, nel corso della seconda metà del Novecento e poi agli albori del ventunesimo secolo, si sia progressivamente verificata non solo l'affermazione di un nuovo spazio metropolitano transnazionale, che ha determinato il superamento delle categorie del margine e del centro, ma anche il significativo cambiamento dei concetti di identità, appartenenza e cultura. La purezza identitaria, etnica e culturale è stata progressivamente sradicata da una realtà sempre più ibrida e meticcia, originata da quei flussi migratori diasporici, che hanno radicalmente ridisegnato la geografia e la storia mondiali, portando all'affermazione di quella che Édouard Glissant definisce come una totalità-mondo. Londra si fa catalizzatore delle differenze culturali e si impone custode esclusiva della formazione e tras-formazione di identità plurime e sincretiche, votate alla fluidità e non già alla staticità della purezza identitaria, ormai desueta. La Londra di Smith e di Kureishi - liberatasi dell'ingombrante fardello dell'alienazione, che grava invece sulle rappresentazioni metropolitane di Naipaul e Selvon, sebbene con esiti talvolta esilaranti - è una città che celebra la diversità, offre lo spazio neutrale per la negoziazione delle differenze e si fa casa per tutti gli outsiders. È il palcoscenico mutevole, il caos-mondo che trasforma, trasformandosi a sua volta, la messinscena delle identità in un "incontro di elementi culturali provenienti da orizzonti assolutamente diversi e che realmente si creolizzano, che realmente si stratificano e si confondono l'uno nell'altro per dar vita a qualcosa di assolutamente imprevisto e di assolutamente nuovo, la realtà creola" (Glissant 1998: 13). Una realtà, in cui le identità si vanno formando e trasformando senza ambire all'essenzialità e alla purezza, ma piuttosto abbandonandosi alla fluidità e alla transitorietà della loro esistenza metropolitana.

\section{Riferimenti bibliografici}

Acroyd, P. (2000). London. The Biography. London: Chatto \& Windus.

Albertazzi, S. (1996). In questo mondo, ovvero quando i luoghi raccontano le storie. Roma: Meltemi.

Ball, J. C. (2004). Imagining London. Postcolonial Fiction and the Transnational Metropolis. Toronto: University of Toronto Press.

Bennett, L. (1986). Colonization in Reverse. In: P. Burnett (Ed.), The Penguin Book of Caribbean Verse in English (pp. 32-33). Harmondsworth: Penguin.

Bhabha, H. (1994). The Location of Culture. London and New York: Routledge. 
Callari Galli, M. (1996). Lo spazio dellincontro. Percorsi nella complessità. Roma: Meltemi.

Chambers, I. (2003). Città senza mappe. In: Paesaggi migratori (pp. 100-122). Roma: Meltemi.

Cuder-Dominguez, P. (2004). Ethnic Cartographies of London in Bernardine Evaristo and Zadie Smith. European Journal of English Studies, 8 (2), 173-188.

Fusillo, M. (2014). Passato presente futuro. In: F. de Cristofaro (Ed.), Letterature comparate (pp. 13-31). Roma: Carocci.

Glissant, É. (1998). Poetica del diverso. Roma: Meltemi.

Hall, S. (1990). Cultural Identity and Diaspora. In: J. Rutherford (Ed.), Identity: Community, Culture, Difference (pp. 234-256). London: Lawrence \& Wishart.

Hall, S. Held, D., \& McGrew, T. (1992). Modernity and its Futures. Cambridge: Polity Press/Open University.

Hall, S. (1996). Minimal Selves. In: H. A. Baker jr, M. Diawara, \& R. H. Lindenborg (Eds.), Black British Cultural Studies. A Reader (pp. 114-119). Chicago: University of Chicago Press.

Hayward, H. (2002). The Enigma of V.S. Naipaul. Sources an Contexts. London: Palgrave Macmillan.

Kureishi, H. (2003). Il Budda delle periferie. Milano: Bompiani.

Lamming, G. (1984). The Pleasures of Exile. London: Allison and Busby.

Lefebvre, H. (1996). Writing on Cities. Oxford: Blackwell.

Manferlotti, S. (1995). La città degli immigrati. Spazio e memoria in Salman Rushdie e Hanif Kureishi. In: C. Pagetti (Ed.), La città senza confini. Studi sull'immaginario urbano nelle letterature di lingua inglese (pp. 300-327). Roma: Bulzoni.

Marcoaldi, F. (2000). Quei denti bianchi e un po' cariati. La Repubblica, 15 Novembre, p. 12.

Naipaul, V. S. (1967). The Mimic Men. London: Andre Deutsch.

- (1988). L'enigma dellarrivo. Milano: Arnoldo Mondadori Editore.

-. (1994). Una via nel mondo. Milano: Adelphi.

- (1999). Un'area di tenebra. Milano: Adelphi.

Rushdie, S. (1991). Patrie Immaginarie. Milano: Arnoldo Mondadori.

Sandhu, S. (2003). London Calling: How Black and Asian Writers Imagined a City. London: Harpercollins.

Selvon, S. (1956). The Lonely Londoners. London: Longman.

—. (1975). Moses Ascending. Oxford: Heinemann.

- (1983). Moses Migrating. Washington: Three Continents Press.

Smith, Z. (2000). Denti Bianchi. Milano: Arnoldo Mondadori Editore.

Stein, M. (1999). Interview with David Dabydeen. Wasafiri, 29, 28-32.

- (2004). Black British Literature. Novels of Transformation. Columbus: The Ohio State University Press.

Tuan, Y. (1977). Space and Place: the Perspective of Experience. Minneapolis: University of Minnesota Press.

Vivan, I. (2002). The Impact of Postcolonial Hybridisation on the Britishness of British Literature. In: S.

Bassi, S. Bertacco, \& R. Bonicelli (Eds), In That Village of Open Doors - Le nuove letterature crocevia della cultura moderna. Atti del I Convegno AISLI. Venezia: Cafoscarina.

Walcott, D. (1981). The Bright Field. In: Selected Poetry (pp. 76-80). London: Heinemann.

Woodcock, B. (1999). I'll Show You Something to Make You Change Your Mind': Post-Colonial Translations of the Streets of London. Kunapipi, 21 (2), 59-62. 